\title{
ESTIMATIVA DE INCERTEZA EXPANDIDA ASSOCIADA À ANÁLISE DE AÇÚCARES REDUTORES PELO MÉTODO DE LANE-EYNON
}

\author{
JUAREZ VICENTE * \\ MARCELO AZEVEDO NEVES ** \\ LUIZ AUGUSTO DA CRUZ MELEIRO ***
}

\begin{abstract}
O objetivo deste trabalho foi estimar a incerteza expandida da análise de açúcares redutores pelo método de Lane-Eynon em amostra de mel. A incerteza de medição foi estimada através do procedimento proposto pelo GUM (INMETRO, 2012), avaliando-se a contribuição percentual de cada etapa do método em estudo e seu impacto na incerteza total por meio do balanço de incerteza. Os resultados mostraram que ambas as condições de análise, utilizando-se balança analítica e bureta de $10 \mathrm{~mL}$ ou balança semi-analítica com bureta de $25 \mathrm{~mL}$, respeitam a função de Horwitz (variação < $4 \%$ ). Entretanto, a variação obtida nessa última condição aproximou-se do limite de $4 \%$. Observou-se que a incerteza da grandeza massa foi insignificante nas condições estudadas. Conclui-se dessa forma que o método Lane-Eynon é metrologicamente confiável e que a incerteza pode ser reduzida quando se aumenta a sensibilidade do instrumento de medição de volume (bureta).
\end{abstract}

PALAVRAS-CHAVE: INCERTEZA EXPANDIDA; MÉTODO DE LANE-EYNON; AÇÚCARES REDUTORES TOTAIS.

* Doutorando em Ciência e Tecnologia de Alimentos, Departamento de Tecnologia de Alimentos, Instituto de Tecnologia, Universidade Federal Rural do Rio de Janeiro (UFRRJ), Seropédica, RJ (e-mail: juarezvd@ gmail.com).

** Doutor em Física, Docente, Departamento de Física, Instituto de Ciências Exatas, UFRRJ, Seropédica, RJ (e-mail: mneves@ufrrj.br).

** Doutor em Engenharia Química, Docente, Departamento de Engenharia Química, Instituto de Tecnologia, UFRRJ, Seropédica, RJ (e-mail: meleiro@ufrrj.br). 


\section{INTRODUÇÃO}

Durante qualquer medição é praticamente impossível obter resultado sem que haja erros (ALBERTAZZI JUNIOR e SOUZA, 2008), porém nenhuma medição seja no comércio, na indústria ou na pesquisa assume significado se não estiver associada à incerteza de medição. Dessa maneira, a incerteza torna-se ferramenta fundamental para o crescimento e inovação tecnológica, possibilitando a promoção da competitividade e do desenvolvimento tecnológico (MENDES e ROSARIO, 2005).

Quando se emite resultado de qualquer espécie é necessário conhecer, ou ao menos estimar, o quanto se pode confiar nele ou o quanto esse resultado se aproxima da realidade. A confiança em tal resultado é obtida mediante conjunto de procedimentos visando favorecer 0 aumento da qualidade da informação.

A aplicação do conceito de incerteza de medição torna-se fundamental para os laboratórios brasileiros que almejam a obtenção de reconhecimento da sua capacidade de realização de ensaios em conformidade com a norma ABNT NBR ISO/IEC 17025 (INMETRO, 2005), a qual estabelece os requisitos gerais necessários para a competência na realização de ensaios e/ou calibrações. Essa norma se aplica a todos os laboratórios que realizam ensaios e/ou calibrações, independente do número de pessoas ou da extensão do escopo das suas atividades, devendo ser utilizada no desenvolvimento do seu sistema de gestão para a qualidade, operações técnicas e administrativas do laboratório.

A utilização da palavra incerteza transmite certo desconforto imediato, ou a sensação de insegurança. Sob o ponto de vista técnico-científico, essa palavra expressa dúvida em relação ao resultado analítico. Independentemente da área de atuação, o estudo e o conhecimento da incerteza de medição associada ao resultado analítico assume extrema importância, em particular quando aplicada à indústria de alimentos. O conhecimento da incerteza possibilita a tomada de decisões quanto às conformidades econômicas, legais e de saúde em produtos passíveis de fiscalização (VICENTE, 2010).

Define-se medição como o "processo de obtenção experimental de um ou mais valores que podem ser, razoavelmente, atribuídos a uma grandeza" (VIM, 2009). Em geral, o resultado da medição representa somente a aproximação ou estimativa do valor verdadeiro e, dessa forma, só será completo quando acompanhado pela incerteza dessa estimativa. A incerteza de medição, no entanto, é definida pelo VIM (2009) como parâmetro não negativo que caracteriza a dispersão dos valores atribuídos a um mensurando com base nas informações utilizadas.

A busca pela melhoria da qualidade de resultados analíticos constitui desafio contínuo e deve estar presente em laboratórios de análise e de calibração. Para a obtenção da acreditação, o laboratório pretendente deve estimar a incerteza expandida dos resultados de análise para os quais almeja o reconhecimento. A incerteza de medição, por ser importante ferramenta no monitoramento da qualidade analítica de ensaios, vem se desenvolvendo muito em diversas áreas do conhecimento, inclusive na de ensaios químicos em alimentos. Contudo, verifica-se que os maiores responsáveis pela produção de conhecimento científico a respeito da estimativa de incerteza são os profissionais da física e das engenharias mecânica e elétrica, visto o grande número de trabalhos sobre o tema publicados nessas áreas (VICENTE, 2010).

O presente trabalho concentra esforços no sentido de contribuir para o aumento de informações relativas a incertezas de medições aplicadas a laboratórios de análise de alimentos, mediante estudo de caso da técnica de análise titulométrica de açúcares devido sua ampla utilização nesses laboratórios. Assim, o objetivo deste trabalho foi estimar a incerteza expandida associada à análise de açúcares redutores pelo método de Lane-Eynon, utilizando amostra de mel como matriz.

\section{MATERIAL E MÉTODOS}

Estudou-se a análise por titulação redox do Teor de Açúcares Redutores (TAR) pelo método de Lane-Eynon, recomendada pela Instrução Normativa (IN) n. 20, de 21 de julho de 1999, do 
Ministério da Agricultura Pecuária e Abastecimento (MAPA).

A escolha do método em questão baseou-se na grande variedade de produtos alimentícios a que essa análise se aplicada e a importância do seu resultado, tanto sob o ponto de vista fiscal, levando em conta o aspecto nutricional, quanto em relação à saúde dos consumidores.

Os experimentos foram realizados no Laboratório Analítico de Alimentos e Bebidas (LAAB), do Departamento de Tecnologia de Alimentos, Instituto de Tecnologia da Universidade Federal Rural do Rio de Janeiro (DTA/IT/UFRRJ), Campus Seropédica (RJ).

\subsection{MATERIAL}

Utilizou-se Determinador de Açúcares Redutores (Redutec ${ }^{\circledR}$ ) Modelo TE - 088 (TECNAL ${ }^{\circledR}$ ) para a realização das análises. Todas as vidrarias (balões volumétricos, pipetas volumétricas e buretas) usadas neste trabalho foram calibradas por laboratórios acreditados pela Coordenação Geral de Acreditação do INMETRO (CGCRE/INMETRO). Os certificados de calibração dos balões e pipetas volumétricas são compostos do valor nominal, valor calibrado, incerteza expandida $(U)$ e fator de abrangência ( $k$ ).

Adquiriu-se a amostra de mel, da flora "Morrão de Candeia", do Apiário da Fazendinha Agroecológica (UFRRJ/Embrapa Agrobiologia/Pesagro-Rio), localizada no campus da UFRRJ em Seropédica (RJ). O padrão de glicose anidra utilizado no experimento para obtenção do Título $(T)$ foi seco em estufa a $105^{\circ} \mathrm{C} \pm 2{ }^{\circ} \mathrm{C}$ por duas horas e resfriado à temperatura ambiente em dessecador para ser utilizado posteriormente no preparo da solução de referência.

\subsection{MÉTODOS}

Adotou-se o procedimento MQT-014 para a determinação do TAR, descrito na IN 20 de julho de 1999 do MAPA (BRASIL, 1999).

\subsubsection{Princípio e descrição do método}

Os glicídios redutores são solubilizados em água, separados por filtração e determinados pelo método Lane-Eynon, sendo os íons cúpricos da solução de Fehling reduzidos quantitativamente, sob ebulição, a óxido cuproso por titulação com solução de açúcar redutor. O ponto final é alcançado quando pequeno excesso do açúcar redutor descolore o indicador azul de metileno.

- Equipamentos - balança analítica; banho-maria; estufa; placa aquecedora.

- Vidraria, utensílios e outros - balões volumétricos de 100, 250 e $1000 \mathrm{~mL}$; bastão de vidro; béquer de $250 \mathrm{~mL}$; bureta de $25 \mathrm{~mL}$; erlenmeyer de $250 \mathrm{~mL}$; funil; papel de filtro qualitativo; pinça de metal; pipeta graduada de $1 \mathrm{~mL}$; pipeta volumétrica de $10 \mathrm{~mL}$; proveta de $50 \mathrm{~mL}$.

- Reagentes - solução de azul de metileno $\left(\mathrm{C}_{16} \mathrm{H}_{18} \mathrm{ClN}_{3} \mathrm{~S} .3 \mathrm{H}_{2} \mathrm{O}\right)$ a $1 \%(\mathrm{~m} / \mathrm{v})$; solução de ferrocianeto de potássio trihidratado $\left(\mathrm{K}_{4}\left[\mathrm{Fe}(\mathrm{CN})_{6}\right] .3 \mathrm{H}_{2} \mathrm{O}\right)$ a $15 \%(\mathrm{~m} / \mathrm{v})$; solução de sulfato de zinco heptahidratado $\left(\mathrm{ZnSO}_{4} \cdot 7 \mathrm{H}_{2} \mathrm{O}\right)$ ou acetato de zinco dihidratado $\left(\left(\mathrm{CH}_{3} \mathrm{COO}\right)_{2} \mathrm{Zn} \cdot 2 \mathrm{H}_{2} \mathrm{O}\right)$ a $30 \%(\mathrm{~m} / \mathrm{v})$.

\section{Etapa 1 - Preparo da Solução de Fehling A}

Dissolver $34,639 \mathrm{~g}$ de sulfato de cobre pentahidratado $\left(\mathrm{CuSO}_{4} \cdot 5 \mathrm{H}_{2} \mathrm{O}\right)$ p.a. e diluir em água destilada a $1000 \mathrm{~mL}$ em balão volumétrico.

\section{Etapa 2 - Preparo da Solução de Fehling B}

Dissolver $173 \mathrm{~g}$ de tartarato duplo de sódio e potássio $\left(\mathrm{C}_{4} \mathrm{H}_{4} \mathrm{NaO}_{6} \cdot 4 \mathrm{H}_{2} \mathrm{O}\right)$ p.a. e $125 \mathrm{~g}$ de hidróxido de sódio $(\mathrm{NaOH})$ p.a. em água. Diluir a $1000 \mathrm{~mL}$ em balão volumétrico. 
Etapa 3 - Preparo da Solução Padrão de Glicose $\left(\mathrm{C}_{\underline{6}} \underline{H}_{12} \underline{\underline{O}} \underline{\underline{\underline{O}}}\right)$

Secar a glicose padrão anidra em estufa a $105^{\circ} \mathrm{C} \pm 2^{\circ} \mathrm{C}$, medir massa próxima de $0,5004 \mathrm{~g}$ $\pm 0,0002 \mathrm{~g} \mathrm{com} k=2,00$ e avolumar para balão volumétrico de $100,037 \mathrm{~mL} \pm 0,020 \mathrm{~mL}$, com $k=2,019$.

Os dados de $U$ e $k$ da massa e volume foram obtidos nos certificados de calibração da balança analítica e do balão volumétrico, respectivamente.

\section{Etapa 4 - Determinação do Título (padronização) da Solução de Fehling}

Colocar a solução padrão na bureta. Transferir com pipeta volumétrica $10,00 \mathrm{~mL}$ de cada uma das soluções de Fehling A e B para erlenmeyer de $250 \mathrm{~mL}$. Adicionar $40 \mathrm{~mL}$ de água e aquecer até ebulição. Gotejar a solução padrão, sem agitação, até quase o final da titulação. Adicionar 1 gota de solução de azul de metileno a 1 \%. Completar a titulação até o descoramento do indicador. A titulação deve ser efetuada sob ebulição e não pode ultrapassar 3 minutos. Calcular o Título da Solução de Fehling de acordo com a equação 1:

$$
T=\frac{V_{p} \cdot m_{g}}{V_{b v}}
$$

Em que:

$V_{p}=$ Volume da solução padrão de glicose;

$m_{g}=$ massa de glicose $(\mathrm{g})$ utilizada no padrão $(\sim 0,5000 \mathrm{~g})$;

$V_{b v}=$ Volume do balão volumétrico $(\sim 100,00 \mathrm{~mL})$.

Prepararam-se os licores de Fehling em erlenmeyer de vidro com capacidade aproximada de $125 \mathrm{~mL}$, utilizando 10,008 $\pm 0,020 \mathrm{~mL}$ com $k=2,042$ para a solução de Fehling A; 10,005 $\pm 0,020 \mathrm{~mL}$ com $k=2,036$ para a solução de Fehling $\mathrm{B}$ e, aproximadamente, $40 \mathrm{~mL}$ de água destilada.

\section{Etapa 5 - Procedimento para Determinação do TAR no mel}

Pesar aproximadamente $25 \mathrm{~g}$ de amostra homogeneizada em béquer de $250 \mathrm{~mL}$. Adicionar $50 \mathrm{~mL}$ de água quente e homogeneizar com bastão de vidro. Transferir para balão volumétrico de $250 \mathrm{~mL}$. Adicionar $5 \mathrm{~mL}$ de solução de ferrocianeto de potássio a $15 \%$ e $5 \mathrm{~mL}$ de solução de sulfato ou acetato de zinco a $30 \%$. Agitar bem e completar o volume do balão. Deixar sedimentar por 15 minutos. Filtrar em papel de filtro seco para erlenmeyer. Colocar na bureta de $25 \mathrm{~mL}$ o filtrado obtido. Pipetar volumetricamente 10,00 mL de solução de Fehling A e 10,00 mL de solução de Fehling B para erlenmeyer de $250 \mathrm{~mL}$. Adicionar $40 \mathrm{~mL}$ de água. Aquecer até ebulição e gotejar a solução da amostra até o líquido sobrenadante ficar levemente azulado. Mantendo a ebulição, adicionar 1 gota de solução de azul de metileno a 1 \% e continuar a titulação sob ebulição até descoloração do indicador. A titulação não poderá ultrapassar 3 minutos, devendo a ebulição ser mantida durante toda a titulação.

\subsubsection{Procedimento para titulação da solução padrão de glicose e para as amostras}

Com a caldeira aquecida, transferiu-se um dos licores de Fehling para o compartimento reacional do Redutec ${ }^{\circledR}$, aguardou-se 1 minuto em ebulição e iniciou-se a titulação com a solução padrão de glicose anteriormente preparada. Quando a coloração azulada da solução (dada pelo íon $\mathrm{Cu}^{+2}$ ) começou a diminuir de intensidade, adicionaram-se duas gotas do indicador azul de metileno e prosseguiu-se a titulação até a descoloração total da solução azul e aparecimento de precipitado de coloração "vermelho tijolo" $\left(\mathrm{Cu}_{2} \mathrm{O}\right)$, indicando o final da titulação. 
quantificação de açúcares redutores. A essa variável se atribui distribuição de probabilidade do tipo triangular (divisor $=\sqrt{6}$ ), devido à tendência (valor calibrado) de mensurá-la conforme descrito no procedimento de análise. Como se trata de balão volumétrico calibrado (dado sem repetição) sua incerteza padrão (equação 3) é obtida no certificado de calibração do balão volumétrico empregado no ensaio com seu respectivo fator de abrangência, $k$.

$$
\begin{gathered}
u\left(d_{a}\right)=\frac{U}{k} \\
u\left(d_{a}\right)=\frac{0,050}{2,023}=0,025 \mathrm{~mL}
\end{gathered}
$$

\subsubsection{Massa da amostra $\left(m_{a}\right)$}

A massa da amostra representa fonte de incerteza de influência direta na quantificação de açúcares redutores. Para essa fonte atribui-se distribuição de probabilidade do tipo retangular (divisor $=\sqrt{3}$ ), pois não há tendência em mensurá-la com valor "mais provável" considerando a última casa decimal da balança analítica ou semi-analítica (dado sem repetição). Obtém-se a incerteza padrão associada a essa fonte por meio do certificado de calibração da balança analítica com seu respectivo fator de abrangência $(k)$.

$$
\begin{gathered}
u\left(m_{a}\right)=\frac{U}{k} \\
u\left(m_{a}\right)=\frac{0,0002}{2,00}=0,0001 \mathrm{~g}
\end{gathered}
$$

\subsubsection{Volume de amostra gasto na titulação $(V a)$}

Para o cálculo da incerteza padrão da fonte volume há a necessidade de avaliá-lo com no mínimo três repetições, possibilitando o cálculo da sua incerteza padrão da média, $\bar{s}$, tratando-se de distribuição normal. Assim sendo, emprega-se a equação 5 que leva em conta $\bar{s}$ e a incerteza padrão do instrumento de medição de volume (bureta), $u_{b}$. Essa incerteza padrão com seu respectivo fator de abrangência, $k$, são obtidos no certificado de calibração da bureta, adotando-se a maior incerteza dos pontos calibrados, no intervalo (faixa de trabalho) do volume lido durante a titulação de determinada amostra.

$$
\begin{gathered}
u\left(V_{a}\right)=\sqrt{\bar{s}^{2}+u_{b}{ }^{2}} \\
u\left(V_{a}\right)=\sqrt{0,04^{2}+0,010^{2}}=0,04 \mathrm{~mL}
\end{gathered}
$$

\subsubsection{Título da solução padrão de glicose (T)}

A fonte de incerteza Título ( $T$ ) apresenta subfontes de incerteza para compor seu resultado. O $T$ (ou seja, a massa em g de glicose padrão necessária para reduzir o $\mathrm{Cu}^{+2}$ da solução de Fehling) é o próprio mensurando. Calcula-se o valor do título de acordo com a equação 6:

$$
T=\frac{m_{p} \cdot V_{p}}{d_{p}}
$$

Em que:

$m_{p}=$ massa (g) de glicose utilizada no preparo de sua respectiva solução padrão;

$V_{p}=$ volume $(\mathrm{mL})$ da solução padrão de glicose gasto na titulação;

$d_{p}=$ diluição da solução padrão de glicose - volume $(\mathrm{mL})$ do balão volumétrico.

\subsubsection{Cálculo dos coeficientes de sensibilidade, $c_{i}$}

Os $c_{i}$ foram obtidos por meio do cômputo da derivada parcial da variável dependente de 
cada uma das fontes de incerteza (variáveis independentes), conforme as equações 7, 8, 9, e 10 para as fontes $d_{a}, T, m_{a}$ e $V_{a}$, respectivamente:

$$
\begin{aligned}
& c_{i}\left(d_{a}\right)=\frac{\partial T A R}{\partial d_{a}}=\frac{T 100}{m_{a} V_{a}} \\
& c_{i}(T)=\frac{\partial T A R}{\partial T}=\frac{d_{a} 100}{m_{a} V_{a}} \\
& c_{i}\left(m_{a}\right)=\frac{\partial T A R}{\partial m_{a}}=\frac{d_{a} T 100}{m_{a}^{2} V_{a}} \\
& c_{i}\left(V_{a}\right)=\frac{\partial T A R}{\partial V_{a}}=\frac{d_{a} T 100}{m_{a} V_{a}^{2}}
\end{aligned}
$$

\subsubsection{Cálculo dos componentes de incerteza}

Realizou-se o cálculo de cada uma das fontes de incerteza com auxílio da equação 11 e de $\operatorname{uTAR}_{\left(x_{i}\right)}$ para as fontes $d_{a}, T, m_{a}$, e $V_{a}$, de acordo com as equações $12,13,14$, e 15, respectivamente:

$$
\begin{gathered}
\operatorname{uTAR}_{\left(x_{i}\right)}=u\left(x_{i}\right) * c_{i}\left(x_{i}\right) \\
u \operatorname{TTAR}_{\left(d_{a}\right)}=u\left(d_{a}\right) * c_{i}\left(d_{a}\right) \\
\operatorname{uTAR}_{\left(d_{a}\right)}=0,025 \mathrm{~mL} * 0,3323383 \mathrm{~mL}^{-1}=0,008308 \\
u T A R_{(T)}=u(T) * c_{i}(T) \\
u \operatorname{TTAR}_{(T)}=0,0001 \mathrm{~g} * 1658,374793 \mathrm{~g}^{-1}=0,165837 \\
u T A R_{\left(m_{a}\right)}=u\left(m_{a}\right) * c_{i}\left(m_{a}\right) \\
u_{T A R_{\left(m_{a}\right)}=} 0,0001 \mathrm{~g} * 55,389718 \mathrm{~g}^{-1}=0,005539 \\
u T A R_{\left(V_{a}\right)}=u\left(V_{a}\right) * c_{i}\left(V_{a}\right) \\
u_{T A R_{\left(V_{a}\right)}=} 0,04 \mathrm{~mL} * 8,267122 \mathrm{~mL}^{-1}=0,330685
\end{gathered}
$$

\subsubsection{Cálculo da incerteza combinada do TAR}

Calculou-se a incerteza combinada do TAR, que consiste no somatório quadrático das incertezas de cada fonte, segundo a equação 16:

$$
\begin{gathered}
u_{c} T A R=\sqrt{u T A R_{\left(d_{a}\right)}^{2}+u T A R_{(T)}{ }^{2}+u T_{A R_{\left(m_{a}\right)}}^{2}+u T A R_{\left(V_{a}\right)}{ }^{2}} \\
u_{c} T A R=\sqrt{(0,008308)^{2}+(0,165837)^{2}+(0,005539)^{2}+(0,330685)^{2}} \\
u_{c} T A R=0,37 \%
\end{gathered}
$$

A Figura 2 ilustra a contribuição (\%) dos componentes de incerteza na incerteza de medição do TAR (balanço de incerteza). 


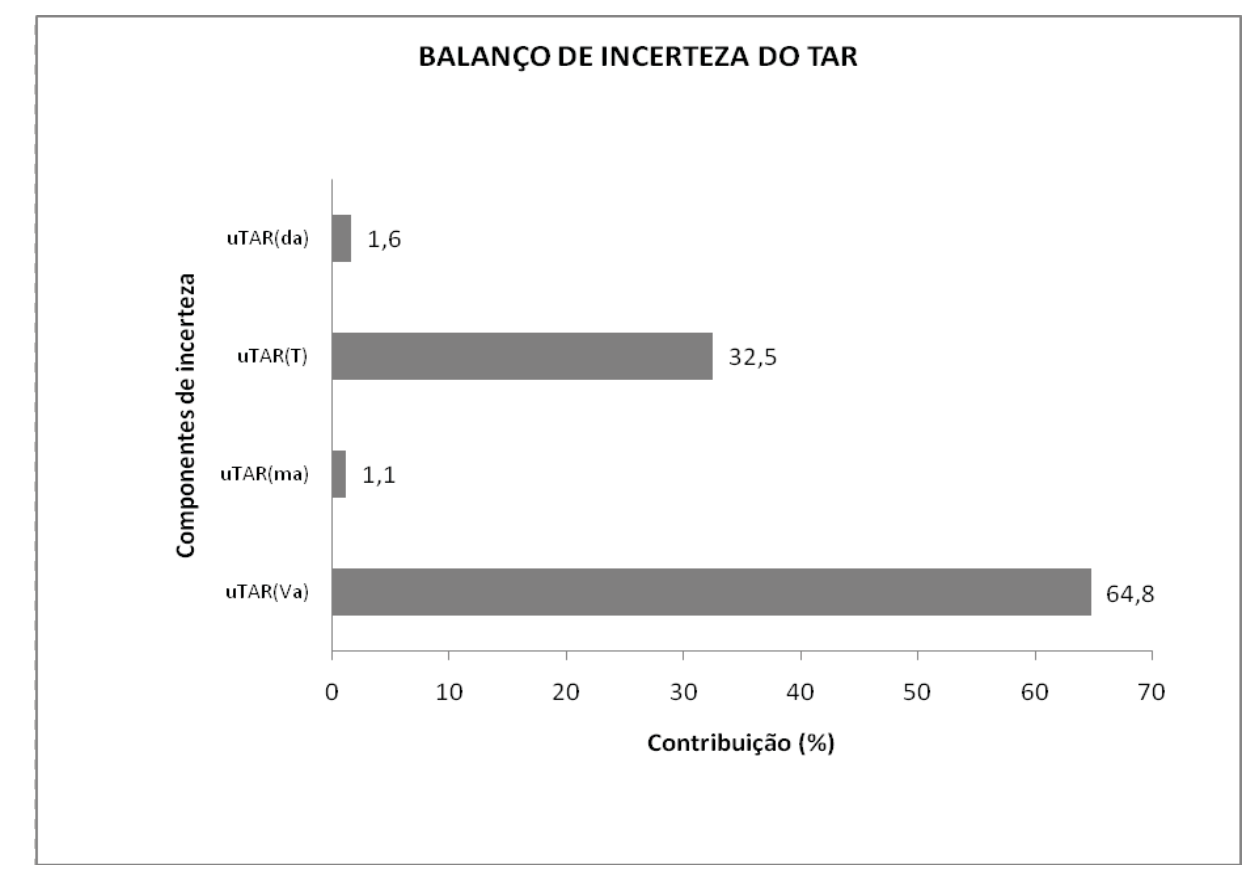

FIGURA 2 - CONTRIBUIÇÕES (\%) DOS COMPONENTES DE INCERTEZA PARA A INCERTEZA DO TAR

\subsubsection{Cálculo dos graus de liberdade efetivos $\left(v_{e f f}\right)$}

Utilizou-se a equação de Wetch-Satterthwaite (Eq. 17) para calcular os graus de liberdade efetivos:

$$
\begin{gathered}
v_{\text {eff }}=\frac{u_{c}^{4}(y)}{\sum_{j=1}^{N} \frac{u_{c}^{4}(y)}{v_{i}}} \\
v_{\text {eff }}=\frac{(0,37)^{4}}{\frac{(0,33068)^{4}}{10-1}}=14,1056 \sim 14
\end{gathered}
$$

\subsubsection{Cálculo do fator de abrangência ( $k$ )}

Empregou-se a função inversa da distribuição $t$-Student, com probabilidade de 95,45 \% $(1-0,9545=0,0455)$ para calcular o fator de abrangência:

$$
\begin{gathered}
k\left(\rho ; v_{\text {eff }}\right) \\
k(0,0455 ; 14)=2,1952
\end{gathered}
$$

3.1.10 Cálculo da incerteza expandida, $U$

$$
\begin{gathered}
U=u_{c} \cdot k \\
U=0,37 \cdot 2,1952=0,8 \%
\end{gathered}
$$

\subsubsection{Representação do resultado de medição}

Obteve-se o resultado da medição por meio da composição do valor do TAR (equação 2) com a incerteza expandida $(\bar{x} \pm U)$ : 


$$
\begin{gathered}
T A R=\frac{d_{a} \cdot T \cdot 100}{m_{a} \cdot V_{a}}=\frac{250,000 \cdot 0,0501 \cdot 100}{1,5000 \cdot 10,05}=83,1 \% \\
\text { Resultado de medição }(\bar{x} \pm U)=(83,1 \pm 0,8 \%)
\end{gathered}
$$

Os valores médios obtidos e expressos como resultados de determinado ensaio são decorrentes do método proposto em função das repetições e dos tratamentos aos quais à amostra foi submetida. Os valores de incerteza não influenciam esse dado, mas são influenciados por seu valor, que é apresentado após o sinal de $\pm(\bar{x} \pm U)$. A incerteza atua sobre esse valor, expressando a flutuação do resultado e, como consequência, a confiabilidade do resultado de medição.

\subsubsection{Título}

De acordo com a Tabela 1 e a Figura 3 verifica-se que a massa da solução padrão de glicose, $m_{p}$, constitui a única variável com potencial para contribuir com o aumento de incerteza do Título.

\begin{tabular}{|c|c|c|c|c|c|c|c|}
\hline Grandeza & $\begin{array}{l}\text { Estimativa } \\
\qquad \text { da } \\
\text { grandeza } \\
\left(x_{i}\right)\end{array}$ & $\begin{array}{c}\text { Distribuição } \\
\text { de } \\
\text { probabilidade }\end{array}$ & $\begin{array}{c}\text { Incerteza } \\
\text { padrão } \\
u\left(x_{i}\right)\end{array}$ & Unidade & $\begin{array}{c}\text { Coeficiente } \\
\text { de } \\
\text { sensibilidade } \\
\qquad\left(C_{\boldsymbol{i}}\right)\end{array}$ & $\begin{array}{c}u T\left(x_{i}\right) \\
(\mathrm{g})\end{array}$ & $\begin{array}{c}\text { Graus de } \\
\text { liberdade } \\
\left(v_{e f f}\right)\end{array}$ \\
\hline$m_{p}$ & 0,5004 & Retangular & 0,0001 & g & 0,100063 & $5,00 \times 10^{-5}$ & 2,000 \\
\hline$V_{p}$ & 10,01 & Normal & 0,010 & $\mathrm{~mL}$ & 0,005002 & $2,46 \times 10^{-5}$ & 2,035 \\
\hline$d_{p}$ & 100,037 & Triangular & 0,010 & $\mathrm{~mL}$ & 0,000501 & $2,48 \times 10^{-6}$ & 2,019 \\
\hline
\end{tabular}

\section{TABELA 1 - CONTRIBUIÇÃO DAS COMPONENTES DA INCERTEZA DO TÍTULO}

O valor percentual de incerteza em relação ao Título obtido no experimento não foi significativo, ficando próximo a $0,40 \%$. Quando esse valor ultrapassa $4 \%$ para essa faixa de concentração $(100 \mathrm{~g} / 100 \mathrm{~g})$ deve-se identificar a fonte ou as fontes que mais contribuem para o aumento da incerteza total e efetuar as ações corretivas para minimizar o problema. Caso o valor da incerteza fosse significativo seria necessário tomar alguma providência em relação à massa do padrão, já que essa fonte é responsável por mais de $60 \%$ da incerteza total da variável Título. Assim, o foco seria o instrumento de medição de massa (balança analítica).

O valor do Título, seus valores de $C_{\boldsymbol{i}}$ e os valores dos componentes de incerteza que compõem a Tabela 1 foram obtidos pela equação 6.

\subsubsection{Título}

$$
T=\frac{0,5004 \cdot 10,01}{100,037}=0,0501 \mathrm{~g}
$$




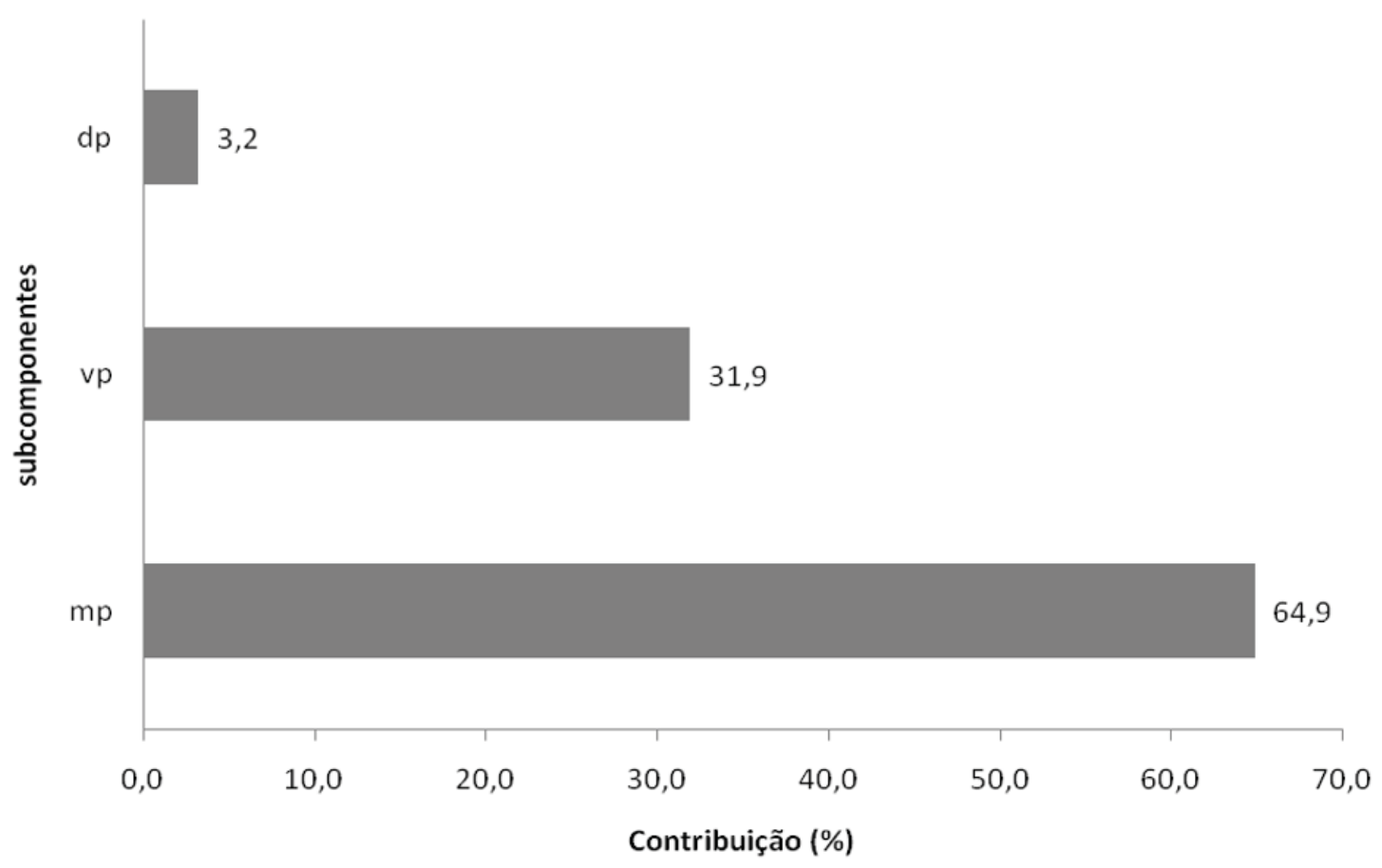

FIGURA 3 - CONTRIBUIÇÕES (\%) DOS COMPONENTES DE INCERTEZA PARA A INCERTEZA DO $T$

\subsubsection{Coeficientes de sensibilidade}

$$
\begin{gathered}
c_{i}\left(m_{p}\right)=\frac{\partial T}{\partial m_{p}}=\frac{V_{p}}{d_{p}}=\frac{10,01}{100,037}=0,100063 \\
c_{i}\left(V_{p}\right)=\frac{\partial T}{\partial V_{p}}=\frac{m_{p}}{d_{p}}=\frac{0,5004}{100,037}=0,005002 \mathrm{~g} \cdot \mathrm{mL}^{-1} \\
c_{i}\left(d_{p}\right)=\frac{\partial T}{\partial d_{p}}=\frac{m_{p} \cdot V_{p}}{d_{p}{ }^{2}}=\frac{0,5004 \cdot 10,05}{100,037^{2}}=0,000501 \mathrm{~g} \cdot \mathrm{mL}^{-1}
\end{gathered}
$$

\subsubsection{Componentes de incerteza}

Efetuou-se o cálculo dos componentes de incerteza da variável $T$ pela equação 20 , que foi obtida multiplicando-se a incerteza padrão da fonte por seu coeficiente de sensibilidade:

$$
\begin{gathered}
u T\left(x_{i}\right)=u\left(x_{i}\right) * c_{i}\left(x_{i}\right) \\
u T\left(m_{p}\right)=\left(\frac{0,0001}{2,00}\right) \mathrm{g} * 0,100063=5 \cdot 10^{-5} \mathrm{~g} \\
u T\left(V_{p}\right)=\left(\frac{0,010}{2,035}\right) \mathrm{mL} * 0,005002 \mathrm{~g} \cdot \mathrm{mL}^{-1}=2,46 \cdot 10^{-5} \mathrm{~g} \\
u T\left(d_{p}\right)=\left(\frac{0,010}{2,019}\right) \mathrm{mL} * 0,000501 \mathrm{~g} \cdot \mathrm{mL}^{-1}=2,48 \cdot 10^{-6} \mathrm{~g}
\end{gathered}
$$




$$
\begin{aligned}
& u_{c} T=\sqrt{u T\left(m_{p}\right)^{2}+u T\left(V_{p}\right)^{2}+u T\left(d_{p}\right)^{2}} \\
& u_{c} T=\sqrt{\left(5.10^{-5}\right)^{2}+\left(2,46.10^{-5}\right)^{2}+\left(2,48.10^{-6}\right)^{2}} \\
& u_{c} T=5,58.10^{-5} \mathrm{~g}
\end{aligned}
$$

\subsubsection{Graus de liberdade efetivos}

Utilizando a equação de Wetch-Satterthwaite (equação 17) para o grau de liberdade efetivo, obteve-se o seguinte resultado:

$$
v_{\text {eff }}=\frac{\left(5,58.10^{-5}\right)^{4}}{\frac{\left(2,46.10^{-5}\right)^{4}}{10-1}} \sim 237
$$

3.1.12.6 Fator de abrangência

Por meio da equação 18, com probabilidade de 95,45 \%, obtém-se o fator de abrangência:

$$
k(0,0455 ; 2,37)=2,0106
$$

\subsubsection{Incerteza expandida \\ $U=u_{c} T \cdot k=5,58 \cdot 10^{-5} \cdot 2,0106=0,0001 \mathrm{~g}$}

\subsubsection{Resultado de medição}

O resultado de medição $(\bar{x} \pm U)$ para a fonte Título foi: $\quad T=(0,0501 \pm 0,0001) \mathrm{g}$

\subsection{DISCUSSÃO}

\subsubsection{TAR na amostra de mel}

Utilizou-se a amostra de mel com o intuito de verificar as variáveis e as contribuições das incertezas em relação ao método e aos componentes, bem como suas influências sobre o resultado final da análise. Escolheu-se essa matriz em função de sua alta concentração de açúcares redutores, condição necessária à boa sensibilidade analítica do método de Lane-Eynon.

Obtiveram-se os dados da concentração de açúcares redutores $(\bar{x})$, sua incerteza expandida, $\boldsymbol{U}$, e seu fator de abrangência, $k(83,1 \% \pm 0,8 \%$, com $k=2,1952)$ associados. Esse valor representa pequena incerteza expandida $(0,98 \%)$, caracterizada como não significativa em relação ao resultado final de análise (teor de açúcares redutores). Isto é justificado pelo fato de que, para a faixa de concentração de $100 \mathrm{~g} / 100 \mathrm{~g}$, os valores estimados para incertezas expandidas aceitos pelo Codex Alimentarius são de até $4 \%$, variando, portanto, com intervalo de confiança aceitável de 96 a 104 g/100 g (SOUZA, 2007). Calcula-se esse dado de acordo com a faixa de concentração por meio da função "trompete" de Horwitz/Thompson (THOMPSON, 2004). Essa função foi determinada 
por estudos colaborativos e mostra que quanto menor a faixa de concentração do analito maior será o percentual de intervalo aceito para a incerteza expandida, chegando a $44 \%$ para concentrações menores que $100 \mu \mathrm{g} / \mathrm{kg}$ (SOUZA, 2007).

Brown et al. (2008) estudaram a estabilidade das medições da produção de mercúrio vaporizado e encontraram incerteza expandida associada à geração na produção da massa de mercúrio vaporizado da ordem de $94,4 \mathrm{ng} \cdot \mathrm{min}^{-1} \pm 2,4 \mathrm{ng} \cdot \mathrm{min}^{-1}$, ou seja, $2,54 \%$ do resultado. A avaliação estendeu-se, ainda, no sentido de verificar nesse percentual qual ou quais das variáveis que mais contribuíram para o aumento do valor de incerteza do resultado. Dessa forma, pode-se determinar quais são as etapas do método em que deve haver maior cautela na execução.

A Tabela 2 apresenta a contribuição de cada componente da expressão matemática do método sobre a incerteza. Assim, pode-se avaliar de maneira quantitativa quais as variáveis que realmente contribuem e quais não contribuem para o aumento da incerteza ou imprecisão do resultado.

\section{TABELA 2 - CONTRIBUIÇÃO DAS ETAPAS PARA A INCERTEZA DO TAR}

\begin{tabular}{ccccccccc}
\hline & $\begin{array}{c}\text { Estimativa } \\
\text { da }\end{array}$ & $\begin{array}{c}\text { Distribuição } \\
\text { Grandeza } \\
\text { grandeza } \\
\left(x_{\boldsymbol{i}}\right)\end{array}$ & $\begin{array}{c}\text { Incerteza } \\
\text { probabilidade }\end{array}$ & $\begin{array}{c}\text { padrão } \\
u\left(x_{\boldsymbol{i}}\right)\end{array}$ & Unidade & $\begin{array}{c}\text { Coeficiente } \\
\text { de } \\
\text { sensibilidade } \\
\left(C_{\boldsymbol{i}}\right)\end{array}$ & $\begin{array}{c}u T\left(x_{\boldsymbol{i}}\right) \\
(\mathrm{g})\end{array}$ & $\begin{array}{c}\text { Graus de } \\
\text { liberdade } \\
\left(v_{\text {eff }}\right)\end{array}$ \\
\hline$d_{a}$ & 250,000 & Triangular & 0,025 & $\mathrm{~mL}$ & 0,332338 & 0,008308 & 2,023 \\
$T$ & 0,0501 & Normal & 0,0001 & $\mathrm{~g}$ & 1658,374793 & 0,165837 & 2,000 \\
$m_{a}$ & 1,5000 & Triangular & 0,0001 & $\mathrm{~g}$ & 55,389718 & 0,005539 & 2,000 \\
$V_{a}$ & 10,05 & Normal & 0,010 & $\mathrm{~mL}$ & 8,267122 & 0,330685 & 2,035 \\
\hline
\end{tabular}

Como se observa na Figura 2, o volume médio gasto da solução de mel na titulação do Licor de Fehling influencia fortemente $(64,8 \%)$ a incerteza expandida do resultado, seguida pelo Título (32,5\%). O volume gasto na titulação da amostra apresenta grande contribuição à incerteza, justificada pelo fato de que a observação do ponto final da reação depende da acuidade visual do analista, vinculando a experiência do analista com a reprodutibilidade do método. Esses dados permitem a avaliação detalhada da metodologia de ensaio em estudo, possibilitando rastrear as etapas com potencial para influenciar a incerteza do método. A partir desses dados evidencia-se que quase $65 \%$ da atenção em relação à confiabilidade do método deve se voltar para a titulação da amostra que, nesse caso, depende da acuidade visual e da experiência do analista. Por se tratar de avaliação sensorial, que depende da sensibilidade humana, sugere-se que sejam testadas novas metodologias capazes de verificar o ponto final da reação de precipitação do $\mathrm{Cu}_{2} \mathrm{O}$, de modo a diminuir a contribuição desse fator sobre o valor da incerteza total na análise de açúcares redutores.

Estudando a incerteza associada ao teor de água de equilíbrio em produtos agrícolas, Silva, Silva e Lima (2005) verificaram teores de 16,4 \% $\pm 3,4 \%$ em sementes de jacarandá-da-bahia, $19,7 \% \pm 5,4 \%$ para angico-vermelho e 15,3\% $\% 1,7 \%$ para óleo-copaíba. Os valores percentuais de incerteza para esses produtos foram de 20,7 \%, 27,4 \% e 11,1\%, respectivamente.

Dias, Camões e Oliveira (2008) avaliaram a concentração (em mg/100 g) de carotenoides (trans $\beta$-caroteno, licopenos totais e luteína) e sua incerteza associada em duas espécies de tomates 
("lido" e "para salada"). Os resultados mostraram que o tomate da variedade "lido" apresentou $1,00 \pm 0,14 \mathrm{mg} / 100 \mathrm{~g}(14 \%)$ para trans $\beta$-caroteno; $8 \pm 2 \mathrm{mg} / 100 \mathrm{~g} \mathrm{(25 \% )} \mathrm{para} \mathrm{licopenos} \mathrm{totais}$ e $0,100 \pm 0,017 \mathrm{mg} / 100 \mathrm{~g} \mathrm{(17 \% )} \mathrm{para} \mathrm{luteína.} \mathrm{Já} \mathrm{a} \mathrm{variedade} \mathrm{"para} \mathrm{salada"} \mathrm{apresentou}$ $0,390 \pm 0,056 \mathrm{mg} / 100 \mathrm{~g}(14,4 \%)$ para trans $\beta$-caroteno; $2,30 \pm 0,57 \mathrm{mg} / 100 \mathrm{~g}(24,8 \%)$ para licopenos totais e $0,080 \pm 0,015 \mathrm{mg} / 100 \mathrm{~g}(19 \%)$ para luteína. Quando comparado com a faixa de incerteza expandida aceitável pelo Codex Alimentarius (de $32 \%$ ) para a concentração de $0,1 \mathrm{mg} / 100 \mathrm{~g}$, os valores estão adequados (SOUZA, 2007). Portanto, é fundamental conhecer as variáveis do método que contribuem para compor a incerteza expandida total e atuar nesses componentes para aperfeiçoar o método de análise, respeitando os valores de critérios de aceitação.

\section{CONCLUSÃO}

Este trabalho possibilitou distinguir os diferentes tipos de incerteza associados à análise de TAR pelo método Lane-Eynon, identificar suas fontes e tratá-las adequadamente. A aplicação do equacionamento referente à metodologia de análise de incerteza permitiu avaliar a influência e a contribuição das variáveis; atribuir fatores adequados; interpretar os resultados obtidos e extrair informações relevantes de dados do certificado de calibração.

Verificou-se que a incerteza expandida associada à análise de TAR pelo método Lane-Eynon não é significativa (<2 \%). Portanto, pode-se concluir que nas condições normais do experimento, o método apresenta baixo percentual de incerteza expandida, o que garante confiabilidade ao resultado.

Constatou-se que embora a componente volume de amostra $\left(V_{a}\right)$ tenha sido a que mais contribuiu para a composição do valor de incerteza do TAR (64,8 \%), não há necessidade de otimizar essa variável porque sua incerteza expandida não atinge nem a metade do limite aceitável. Já o impacto da variável Título é menor (31,9\%).

Pode-se considerar que o volume da amostra $\left(V_{a}\right)$ representa a única variável que pode contribuir de forma significativa para a incerteza das variáveis do TAR. Esse resultado evidencia que o instrumento de medição de volume - neste estudo a bureta - é a vidraria responsável pela maior contribuição para o aumento de incerteza da metodologia.

Pode-se concluir que, com analistas bem treinados, o método titulométrico de oxi-redução de análise de açúcares Lane-Eynon constitui técnica bastante confiável nas condições de utilização descritas no método.

Os procedimentos metrológicos aplicados nesta pesquisa contribuíram para o aumento de informações relacionadas à incerteza do ensaio titulométrico de açúcares redutores pelo método Lane-Eynon.

\section{ABSTRACT \\ ESTIMATION OF EXPANDED UNCERTAINTY ASSOCIATED TO ANALYSIS OF REDUCING SUGARS BY LANE-EYNON METHOD}

The aim of this work was to estimate the expanded uncertainty of the analysis of reducing sugars by LaneEynon method in honey sample. The measurement uncertainty was estimated through the procedure proposed by GUM (INMETRO, 2012), evaluating the percentage of contribution of each step of the method and its impact over total uncertainty through uncertainty balance. The results showed that both analyses conditions, the one using analytical balance with burette of $10 \mathrm{~mL}$ as that using semi-analytical balance with burette of $25 \mathrm{~mL}$, respect the Horwitz function (< $4 \%$ variation). However, the variation obtained in this last condition comes very close to the limit of $4 \%$. It was noted, that the uncertainty of mass measurement was not significant in these studied conditions. It was concluded that this is a metrologically reliable method and that the uncertainty can be reduced with the increase of sensibility of volume measurement instrument (burette). 


\section{REFERÊNCIAS}

1 ALBERTAZZI JUNIOR, A.G.; SOUZA, A.R. Fundamentos de metrologia científica e industrial. Barueri, SP: Manole, 2008.

2 BRASIL. Ministério da Agricultura, Pecuária e Abastecimento. Instrução Normativa n. 20 de 21 de julho de 1999. Oficializa os métodos físico-químicos para controle de produtos cárneos e seus ingredientes, sal e salmoura. MQT-014. Diário Oficial [da] República Federativa do Brasil, Brasília, 27 de julho de 1999. Seção 1, p.10.

3 BROWN, A.S.; BROWN, R.J.C.; CORNS, W.T.; STOCKWELL, P.B. Establish SI traceability for measurement of mercury vapour. The Analyst, v.133, p.946-953, 2008. DOI: 10.1039/b803724h. The Royal Society of Chemistry, 2008.

4 DIAS, M.G.; CAMÕES, M.F.G.F.C.; OLIVEIRA, L. Uncertainty estimation and in-house method validation of HPLC analysis of carotenoids for food composition data production. Food Chemistry, v. 109, p. 815-824, 2008.

5 Instituto Nacional de Metrologia Qualidade e Tecnologia (INMETRO). Requisitos gerais para a competência de laboratórios de ensaio e calibração: ABNT NBR ISO/IEC 17025. 2. ed. Rio de Janeiro, 2005. 31 p.

6 Instituto Nacional de Metrologia Qualidade e Tecnologia (INMETRO). Guia para a expressão de incerteza de medição: avaliação de dados de medição - GUM 2008. Duque de Caxias (RJ), 2012. 141 p.

7 MENDES, A.; ROSARIO, P.P. Metrologia e incerteza de medição. São Paulo: Editora EPSE, 2005.

8 SILVA, W.P.; SILVA, C.D.P.S.; LIMA, A.G.B. Incerteza na determinação do teor de água de equilíbrio de produtos agrícolas. Revista Brasileira de Produtos Agroindustriais, Campina Grande, Especial, v.7, n.2, p.159-164, 2005.

9 SOUZA, S. V. C. Procedimentos para validação intralaboratorial de métodos de ensaio: deliniamento e aplicabilidade em análise de alimentos. 2007. 297 f. Tese (Doutorado em Ciências de Alimentos), Faculdade de Farmácia, Universidade Federal de Minas Gerais, Belo Horizonte, 2007.

10 THOMPSON, M. The amazing Horwitz function. Royal Society of Chemistry, 17 AMC Technical Brief. v.2, n.17, Jul, 2004. Disponível em: http://www.rsc.org/images/horwitz-function-technical-brief-17_tcm18-214859.pdf. Acesso em: 29/04/2013.

11 VICENTE, J. Determinação da incerteza expandida associada à análise de açúcares redutores pelo método de Lane-Eynon. 2010. 79 f. Dissertação (Mestrado em Ciência e Tecnologia de Alimentos) - Instituto de Tecnologia, Universidade Federal Rural do Rio de Janeiro, Seropédica, 2010.

12 VIM. Vocabulário internacional de metrologia: conceitos fundamentais e gerais e termos associados. 1. ed. LusoBrasileira. Rio de Janeiro: INMETRO, 2012. 